\title{
Stability of Interfaces with Mesh Refinement
}

\author{
By Marsha J. Berger*
}

\begin{abstract}
We study the stability of mesh refinement in space and time for several different interface equations and finite-difference approximations. First, we derive a root condition which implies stability for the initial-boundary value problem for this type of interface. From the root condition, we prove the stability of several interface equations using the maximum principle. In some cases, the final verification steps can be done analytically; in other cases, a simple computer program has been written to check the condition for values of a parameter along the boundary of the unit circle. Using this method, we prove stability for Lax-Wendroff with all the interface conditions considered, and for Leapfrog with interpolation interface conditions when the fine and coarse grids overlap.
\end{abstract}

1. Introduction. In solving a hyperbolic partial differential equation by finite-difference techniques, a grid with variable-mesh spacing is required to accurately resolve the solution over the entire domain with a minimum number of grid points. One simple way to effect this is by an abrupt mesh refinement in space, where the mesh spacing on one side of the interface is an integral multiple of the mesh width on the other side. A drawback of this approach is that for stability reasons, the time step $\Delta t$ on the coarser side is limited by the smaller mesh width. This has motivated the use of mesh refinement in time and space, where the time step on the coarser side of the interface is also an integral multiple of the time step on the finer side of the interface, though possibly a different integer than for the spatial refinement.

This situation arises, for example, in the following problem. Consider the scalar equation $u_{t}=u_{x},-\infty<x<\infty$, which will be approximated using a coarse grid for $x<0$ and a fine grid for $x>0$. As illustrated in Figure 1.1, the fine grid is refined by a factor of 4 in space and time over the coarse grid. If the Lax-Wendroff difference scheme,

$$
v_{\nu}^{m+1}=v_{\nu}^{m}+\frac{\lambda}{2}\left(v_{\nu+1}^{m}-v_{\nu-1}^{m}\right)+\frac{\lambda^{2}}{2}\left(v_{\nu+1}^{m}-2 v_{\nu}^{m}+v_{\nu-1}^{m}\right)
$$

is used on both the coarse and fine grids, one way to match over the interface is as follows. To get $v_{0}^{1}$ on the coarse mesh, the Lax-Wendroff scheme is applied at $v_{0}^{0}$, taking $v_{1}^{0}=u_{4}^{0}$ for the missing value to the right. The values $u_{0}^{k}, 1 \leqslant k \leqslant n-1$, are

Received August 18, 1983; revised October 30, 1984.

1980 Mathematics Subject Classification. Primary 65M10.

*Supported in part by the National Science Foundation under Grant No. MCS77-02082, by Department of Energy Contract No. DEAC0276ER03077-V, and by the National Aeronautics and Space Administration under NASA Contract No. NAS1-17070, while the author was in residence at the Institute for Computer Applications in Science and Engineering, NASA Langley Research Center, Hampton, VA 23665. 
obtained by linear interpolation in time using $v_{0}^{0}$ and $v_{0}^{1}$, or equivalently, $u_{0}^{0}$ and $u_{0}^{4}$ (see Section 4 for another method). These values are then used in the Lax-Wendroff formulas on the refined side. This procedure is now repeated for each coarse time step. The numerical scheme is then completely defined.

In this paper, we present the first stability proofs for the case of an interface where the mesh is refined in both space and time. Our result, based on the Kreiss [16] and Gustafsson, Kreiss and Sundström [14] stablity theory for the initialboundary value problem, begins with the derivation of a root condition which is equivalent to stability for this case. Using this, we can prove stability of the Lax-Wendroff difference scheme with several different interface conditions. Oliger [17] has an unstable example of the nondissipative Leapfrog scheme with interpolatory interface conditions in the case of mesh refinement by an even integer. We can extend our method of proof to the case of mesh refinement in time and space in which the fine and coarse grid points do not coincide, but rather where the two meshes overlap a bit. The stability of Lax-Wendroff is (not surprisingly) preserved. Moreover, the spatial interpolation introduced into the interface equations by the overlapping meshes is enough to prove stability for the Leapfrog scheme as well.

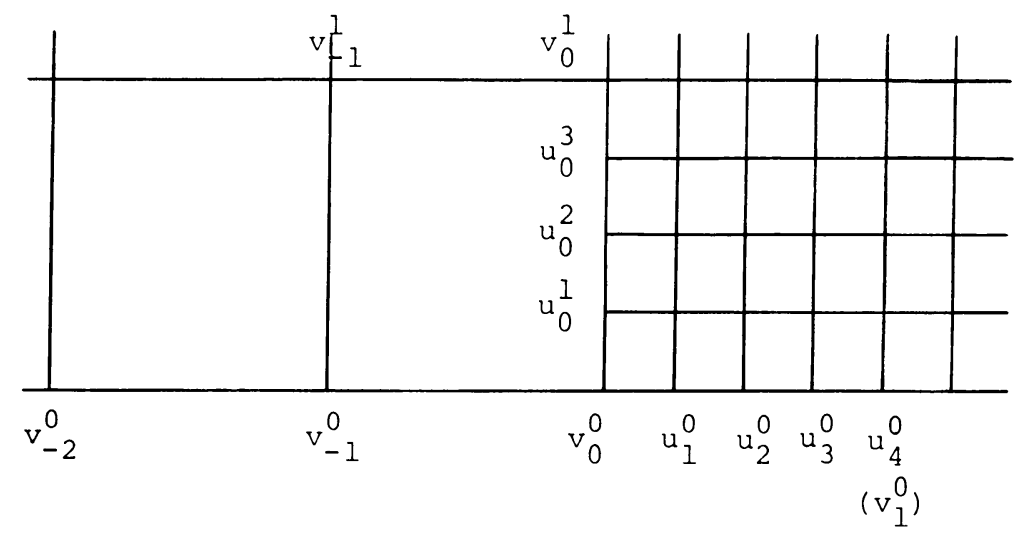

FIGURE 1.1

Interface with mesh refinement in space and time by a factor of 4

The stability of an interface under an abrupt mesh refinement in space only has previously been considered by several authors. Ciment [8] has proved the stability of the Lax-Wendroff difference scheme under very general interface conditions. In [9], he extended his stability results to the case of an interface where a dissipative difference scheme was used on at least one side of the interface. Browning, Kreiss and Oliger [7] considered the Leapfrog scheme under an overlapping mesh refinement, and proved stability using interface equations specifying continuity of the solution and its first derivative. Goldberg and Tadmor [11] consider stability in the case of translatory boundary equations for the outflow variables. Starius [21] proves stability for the slightly different problem of overlapping grids with the same mesh widths in time and space, where interpolation formulas unite the two computations. None of these results apply in the case of mesh refinement in time and space. In fact, Trefethen [23] shows by example that complicated boundaries and interfaces can be unstable even when all formulas involved are dissipative. 
There are certainly other important properties of interfaces worth considering besides stability. The conservation properties of an interface can be crucial if one is computing a discontinuous solution (Berger [3]). Vichnevetsky and Bowles [24] and Trefethen [23] have studied reflection and transmission properties of an interface.

Although the theory lags, computations have been successfully done for many years. Starius does simulations using two overlapping meshes in different coordinate systems. This is becoming more common in the aircraft industry for problems with complex geometries [6]. More recent computations of this type have been done by $\mathrm{B}$. Kreiss [15] and by Reyna [20]. Many computations have been done using mesh refinement in time and space, in both one dimension (Bolstad, [5]) and two dimensions (Gropp, [12]; Berger and Oliger, [4]). These computations use locally uniform meshes with grid spacings $h_{1}, h_{2}$, and time steps $k_{1}$ and $k_{2}$ chosen appropriately for each grid. This is usually done so that the mesh ratio $\lambda=k_{1} / h_{1}=$ $k_{2} / h_{2}$ is constant on both grids. This mesh refinement technique has proven to be very efficient.

In the rest of this section we will briefly recall the stability theory of Kreiss [16] and Gustafsson, Kreiss and Sundström [14] that we will use here. In Section 2, we derive what we call the root condition for stability of an interface under mesh refinement in space and time, where the interface equations come from interpolation in time. For this derivation, we assume the finite-difference scheme on both sides of the interface has a three-point stencil, so that only one point in space needs a special boundary equation. We are thus led to the root condition which unstable eigensolutions (if there are any) must satisfy. In Section 3, we make use of the maximum principle to show that the root condition cannot hold for the roots of the LaxWendroff difference operator, and thus prove that Lax-Wendroff with interpolation interface conditions is stable. In Section 4, we briefly repeat the same type of derivation as in Section 2, but where the interface equations come from the Coarse Mesh Approximation Method (Ciment, [9]), rather than interpolation. In this case, the root condition is more complicated, and we resort to the computer to verify that for the case of Lax-Wendroff, it cannot hold. Our use of the maximum principle, however, reduces the numerical verification to values of a parameter on the boundary of the unit circle. The use of the computer in checking stability is not new; see, for example, Pereyra, Proskurowski and Widlund [19], Coughran [10] or Gustafsson [13]. In Coughran's work, it was necessary to check stability for a range of values within the unit circle. Gustafsson has reduced the problem to checking values near the boundary of the unit circle. Neither of these approaches, however, is easily adapted to cover the case of mesh refinement in time and space. In Section 5, we again consider the case of interpolation in time for the interface conditions, but this time, where the grids do not meet exactly at a grid point. If the one-dimensional grids overlap, interpolation in space is also necessary to determine the interface solution values. This mimics more closely the situation in two-dimensional mesh refinement calculations. It is no surprise that this case is stable if the nonoverlapping case is. In fact, the additional interpolation in space adds enough smoothing to stabilize the unstable Leapfrog configuration with interpolation interface equations when the grids do match up evenly. This has previously been observed by Reyna [20] for the case of mesh refinement in space only. 
The stability theorems of Kreiss [16] and Gustafsson, Kreiss and Sundström [14] can be stated roughly as follows. Consider a Cauchy stable difference scheme for the constant coefficient hyperbolic initial-boundary value problem

$$
u_{t}=A u_{x}+B u+F(x, t), \quad 0 \leqslant x \leqslant \infty,
$$

where $A, B$ are constant $n$ by $n$ matrices, $u$ and $F \in \mathbf{R}^{n}$. Denote the solution of the difference scheme by $u_{\nu}^{m} \approx u(\nu h, m k)$. We will look for normal mode solutions of the form

$$
u_{\nu}^{m}=z^{m} \phi_{\nu}
$$

where $\phi$ is the solution to the characteristic equation of the difference scheme. This results from a discrete Laplace transform in time and the subsequent recurrence relation in space. The solution $\phi$ is thus a linear combination of solutions

$$
\phi_{\nu}=\sum_{\left|\kappa_{j}\right| \leqslant 1} P(\nu, j) \kappa_{j}^{\nu}(z), \quad \nu \geqslant \nu_{0},
$$

where the $\kappa_{j}=\kappa_{j}(z)$ are the distinct roots of the characteristic equation, and the degree of the polynomial $P$ with respect to $\nu$ is one less than the geometric multiplicity of the corresponding root $\kappa_{j}$. An important proposition of Gustafsson, et al. [14, Lemma 5.2, p. 660] shows that for any $z$ with $|z|>1$, there are $n l$ linearly independent solutions with $|\kappa|<1$ and $n r$ solutions with $|\kappa|>1$, where $l$ and $r$ are the number of points in the difference stencil to the left and right of center respectively. For the solution $\phi$ to be in $l_{2}$, the sum in (1.2) is taken over those roots $\left|\kappa_{j}\right|<1$, and includes roots $\left|\kappa_{j}\right|=1$ only if they are roots which approach the boundary of the unit circle from the inside as $|z| \rightarrow 1$ from outside the unit circle. If there are no solutions of the form (1.1) and (1.2) with $|z|>1$ and $\left|\kappa_{j}\right|<1$ or $|z|=1$ and $\left|\kappa_{j}\right| \leqslant 1$ then the difference approximation is stable.

In the case of an explicit, two-level dissipative scheme applied to $u_{t}=A u_{x}$, stability implies the estimate (Kreiss, [16])

$$
\|u(\cdot, t)\|_{x} \leqslant K_{T}\|u(\cdot, 0)\|_{x} .
$$

Under certain circumstances, results of Osher [18] give the same estimate for nondissipative models also. But for general nondissipative or multi-level schemes, we only get the weaker estimate (Gustafsson, Kreiss and Sundström, [14])

$$
\begin{aligned}
\left(\frac{\alpha-\alpha_{0}}{1+\alpha k}\right) & \sum_{\nu=0}^{l-1}\left\|e^{-\alpha t} v_{\nu}\right\|_{t}^{2}+\left(\frac{\alpha-\alpha_{0}}{1+\alpha k}\right)^{2}\left\|e^{-\alpha t} v\right\|_{x, t}^{2} \\
& \leqslant K_{0}^{2}\left[\left(\frac{\alpha-\alpha_{0}}{1+\alpha k}\right) \sum_{\nu=0}^{l-1}\left\|e^{-\alpha(t+k)} g_{\nu}\right\|_{t}^{2}+\left\|e^{-\alpha(t+k)} F\right\|_{x, t}^{2}\right]
\end{aligned}
$$

for some $\alpha_{0}$ and all $\alpha>\alpha_{0}$, and for all sufficiently small $k$. In this definition $t=m k$, $g$ is the boundary data, and $F$ is a forcing function. In (1.4), the norm of the solution in the interior of the domain is taken in both space and time. Recent results of Gustafsson [13] give conditions so that an $l_{2}$-estimate exists for nondissipative schemes as well. See Trefethen ([22] and [23]) for a more detailed exposition of the stability theory for initial-boundary value problems. 
The last point we make concerns the so-called folding trick (Ciment, [9]). Consider a scalar problem with an interface at $x=0$, with one difference scheme on the left and a possibly different difference scheme on the right, and some interface equations. The stability of the combined scheme can be determined by folding the left side along the line $x=0$, thus transforming the problem into an equivalent 2 by 2 system of equations for $x \geqslant 0$, with boundary conditions determined by the interface equations. Then, we can apply the initial-boundary value theory. For example, to approximate $u_{t}=a u_{x}$, we can approximate the equivalent system

$$
\left(\begin{array}{l}
u_{1} \\
u_{2}
\end{array}\right)_{t}=\left(\begin{array}{cc}
a & 0 \\
0 & -a
\end{array}\right)\left(\begin{array}{l}
u_{1} \\
u_{2}
\end{array}\right)_{x}
$$

where $u_{1}(x)=u(x)$ and $u_{2}(x)=u(-x)$ for $x>0$. It is unnecessary to actually go through the folding before applying the initial-boundary value problem theory, especially since it tends to confuse the notation. We point out that by using this and definition (1.4), the solution at the interface will receive special weight that does not appear in definitions of Cauchy stability. Trefethen [22] has shown that if one applies the folding trick to the Cauchy problem for $u_{t}=u_{x}$, and applies Leapfrog at all grid points including the interface point, the result has a generalized eigenvalue.

2. Derivation of Root Condition for 3-Point Difference Schemes. In this section we derive the root condition for stability of an interface under mesh refinement in space and time. Consider the scalar wave equation, $u_{t}=u_{x}$, with the coarse grid on the left of the interface $x=0$, and the fine grid on the right. Later, we will consider the case $u_{t}=-u_{x}$, or equivalently, the case when the fine and coarse grid locations are reversed. Let the coarse grid be refined by an arbitrary integral factor $n$. An equal refinement factor in time and space is not an essential part of this derivation, but for simplicity we will assume it. We will assume that the difference operator applied on both the coarse and fine grids has a three-point stencil, so that only at the one point where the stencil cannot be applied do we need a special boundary condition. In this derivation, it is not necessary that the difference operator on the coarse and fine grids be the same.

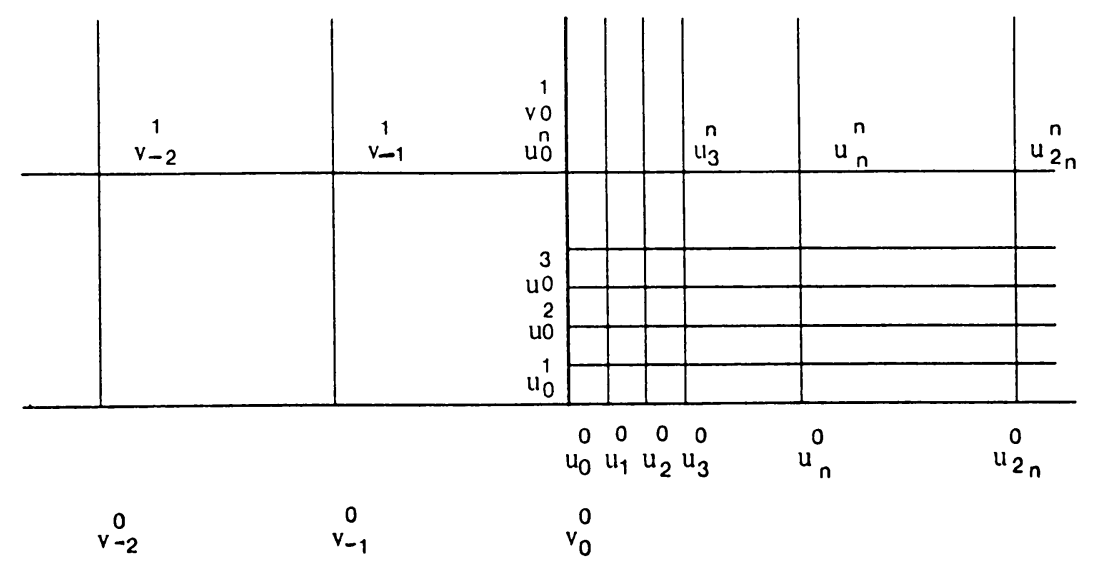

Figure 2.1

Interface with mesh refinement in space and time 
The situation we model is illustrated in Figure 2.1. In Figure 2.1, we have drawn the intermediate time steps on the fine grid, but in fact we can view the difference scheme on the right as being one application of $Q^{n}$, where $Q$ is a three-point finite-difference operator with mesh ratio $\lambda$. The GKS theory says to look for modes which grow geometrically in time. Let this factor in time be $z^{n}$, instead of $z$ in (1.1). The difference equations form a three-term recurrence relation for the spatial part $\phi$ in (1.2), which has two roots. By the previously mentioned proposition of Gustafsson, et al. there are as many roots $\kappa_{j},\left|\kappa_{j}\right|<1$, of the characteristic equation as there are points to the left of center in the stencil for the difference operator. Correspondingly, there are as many roots with $\left|\kappa_{i}\right|>1$ as there are points to the right. For example, for the Lax-Wendroff difference operator,

$$
v_{\nu}^{m+1}=v_{\nu}^{m}+\frac{\lambda}{2}\left(v_{\nu+1}^{m}-v_{\nu-1}^{m}\right)+\frac{\lambda^{2}}{2}\left(v_{\nu+1}^{m}-2 v_{\nu}^{m}+v_{\nu-1}^{m}\right)
$$

the characteristic equation is

$$
z=1+\frac{\lambda}{2}\left(\kappa-\kappa^{-1}\right)+\frac{\lambda^{2}}{2}\left(\kappa-2+\kappa^{-1}\right)
$$

The two roots of (2.1) are

$$
\kappa_{ \pm}=\frac{z-1+\lambda^{2} \pm \sqrt{(z-1)^{2}+\lambda^{2}(2 z-1)}}{\lambda^{2}+\lambda} .
$$

The root $\kappa_{-}$is inside the unit circle, $\kappa_{+}$is outside. Henceforth, we call the roots $\kappa_{\text {in }}$ and $\kappa_{\text {out }}$.

Since for 3-point difference schemes, there is only one root inside the unit circle, the normal modes (1.1) and (1.2) take a particularly easy form. Therefore, on the coarse grid to the left of the interface we look for the modes $v_{\nu}^{m}=\operatorname{constant}\left(z^{n}\right)^{m} \tau^{\nu}$, where for simplicity we will normalize the constant to 1 . We use $\tau$ for the root of the resolvent equation for $x<0$. Since the coarse grid points are numbered with negative indices, for the solution $v_{\nu}$ to be in $l_{2}$ we want the root $\tau$ for which $|\tau| \geqslant 1$.

On the right of the interface, $n$ applications of a difference operator $Q$ has a stencil $n$ points to the left of center. By the lemma above, there must be $n$ roots of the characteristic equation inside the unit circle. Let $\omega_{j}, j=0,1, \ldots, n-1$ be the $n$ roots of unity; $z \omega_{j}$ are then the $n$ roots of $z^{n}$. For every value $z \omega_{j}$ there is a corresponding root $\kappa_{j}$ of the difference scheme. Each such mode $\kappa_{j}$ satisfies the requirement that after one global repetition of the difference equations, or $n$ steps on the fine grid, the solution in space is multiplied by $z^{n}$. There are $n$ such modes $\kappa_{j}$, and since the mapping from the $z_{j}=z \omega_{j}$ with modulus $>1$ to $\kappa_{j}$ inside the circle is one to one, the $\kappa_{j}$ are distinct. The general solution represented on the fine grid must therefore be of the form

$$
u_{\nu}^{m}=\sum_{j=0}^{n-1} \rho_{j} \kappa_{j}^{\nu}\left(z \omega_{j}\right)^{m}
$$

where the $\rho_{j}$ are constants. This is illustrated in Figure 2.2. 


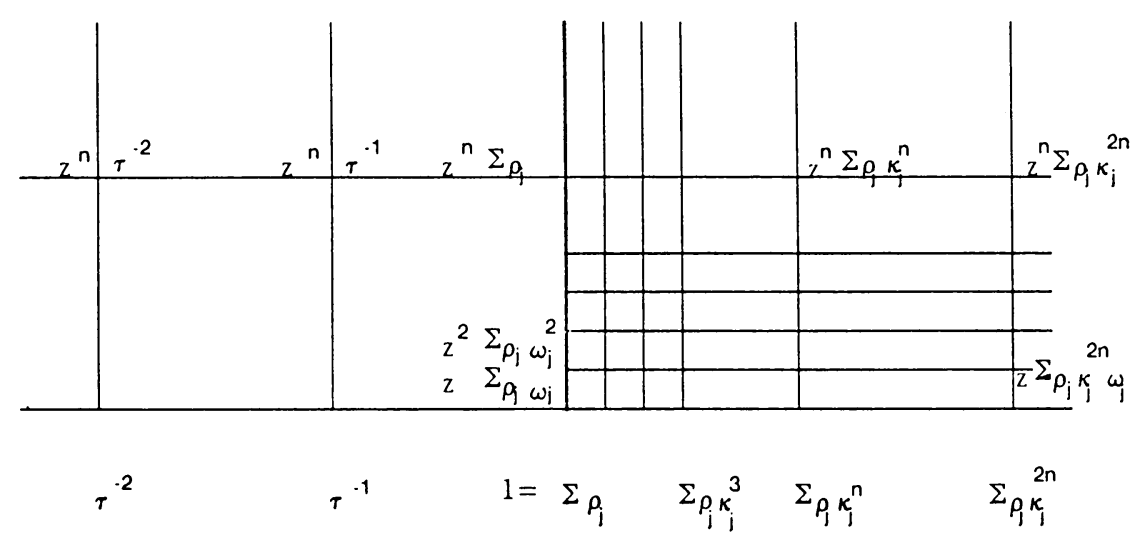

FIGURE 2.2

Normal modes of the solution

We point out that the solution on either side of the interface must take the form of the normal modes at all grid points up to and including the boundary point. On the left, we have a three-point scheme which can be applied at all grid points but $v_{0}$, where we must specify one boundary condition. Similarly, at each fine grid time step we can apply the difference operator $Q$ at each point $u_{\nu}, \nu \geqslant 1$, and specify $n$ conditions for the $u_{0}^{i}, i=1, \ldots, n$.

We now examine the interface conditions that these modes must satisfy. The first requirement is that the grid point at the interface belong to both the coarse grid and the fine grid, $v_{0}^{1}=u_{0}^{n}$, or

$$
\sum_{j} \rho_{j}=1
$$

Next, to compute $v_{0}^{1}$ we use a coarse mesh approximation, i.e., we use the grid point $u_{n}^{0}$ for $v_{1}^{0}$. Since we are looking for normal modes, and $v_{0}^{0}, v_{-1}^{0}$, and $z$ are already determined, this means that the point $u_{n}^{0}$ must fit into the normal mode of the coarse grid as the point $v_{1}^{0}$, or

$$
\tau=\sum_{j} \rho_{j} \kappa_{j}^{n}
$$

For the remaining $n-1$ grid points $u_{0}^{k}$ at the interface we will use linear interpolation in time,

$$
u_{0}^{k}=v_{0}^{0}+\frac{k}{n}\left(v_{0}^{1}-v_{0}^{0}\right), \quad 1 \leqslant k \leqslant n-1,
$$

giving us the equations

$$
1+\frac{k}{n}\left(z^{n}-1\right)=z^{k} \sum_{j} \rho_{j} \omega_{j}^{k}, \quad k=1, \ldots, n-1 .
$$


Equations (2.3) and (2.6) together give $n$ conditions that the constants $\rho_{j}, j=$ $0, \ldots, n-1$, must satisfy. We can write this as the linear system $W \rho=b$,

$$
\left.\begin{array}{cccccc}
1 & 1 & 1 & \cdots & 1 \\
\omega_{0} & \omega_{1} & \omega_{2} & \cdots & \omega_{n-1} \\
\omega_{0}^{2} & \omega_{1}^{2} & \omega_{2}^{2} & \cdots & \omega_{n-1}^{2} \\
\cdot & \cdot & \cdot & \cdot & \cdot \\
\omega_{0}^{n-1} & \omega_{1}^{n-1} & \omega_{2}^{n-1} & \cdots & \omega_{n-1}^{n-1}
\end{array}\right)\left(\begin{array}{c}
\rho_{0} \\
\rho_{1} \\
\rho_{2} \\
\cdot \\
\rho_{n-1}
\end{array}\right)
$$

Since the matrix $W$ on the left-hand side is a Vandermonde matrix, and the roots of unity are distinct, we can solve uniquely for the $\rho_{j}$. Furthermore, if we normalize the matrix $W$, defining $F=W / \sqrt{n}$, then $F$ is unitary, $F^{*} F=I$.

We then solve for $\rho=F^{*} b$ to get

$$
\rho_{j}=\frac{1}{n}\left[\sum_{k=0}^{n-1}\left(\frac{\bar{\omega}_{j}}{z}\right)^{k}\right]+\frac{\left(z^{n}-1\right)}{n^{2}}\left[\sum_{k=0}^{n-1} k\left(\frac{\bar{\omega}_{j}}{z}\right)^{k}\right] .
$$

The last two terms in (2.7) can be summed explicitly. In particular, we use the relation

$$
\sum_{k=0}^{n-1} k r^{k}=\frac{r}{(1-r)^{2}}\left[r^{n}(n-1)-n r^{n-1}+1\right] .
$$

Summing and simplifying finally gives the expression for the constants

$$
\rho_{j}=\frac{\left(z^{n}-1\right)^{2}}{\left(z-\bar{\omega}_{j}\right)^{2}} \frac{\bar{\omega}_{j}}{n^{2} z^{n-1}} .
$$

There is still one equation from the interface conditions, (2.4), that we have not used. Substituting the expression for the $\rho_{j}$ into (2.4) gives the root condition

$$
\tau=\sum_{j=0}^{n-1}\left(\frac{z^{n}-1}{z-\bar{\omega}_{j}}\right)^{2} \frac{\bar{\omega}_{j}}{n^{2} z^{n-1}} \kappa_{j}^{n} .
$$

Let us summarize at this point. If there are eigenvalues or generalized eigenvalues that satisfy both the difference equations and the interface equations, they must satisfy (2.9). If (2.9) has an $l_{2}$ solution with $|z|>1$, or a generalized $l_{2}$ solution for $|z|=1$, then the difference equations are unstable. In the next section, we will show that for any two-step three-point dissipative scheme, in particular, using the LaxWendroff difference operator on both the fine and coarse grids, linear interpolation for the interface equations is stable. 
3. Stability of Lax-Wendroff. In Section 2, we derived the root condition for interpolation interface conditions assuming the difference scheme could be applied at all points up to, but not including, the interface. In this section, we show that for the particular choice of the Lax-Wendroff difference scheme on both the fine and coarse grids, the root condition cannot hold. In fact, this is true for any three-point two-step explicit finite-difference scheme. For multi-level dissipative schemes, an additional condition must be satisfied. Since the stability proof for the special case of Lax-Wendroff is so simple and straightforward, we prove it separately before considering the more general dissipative case.

THEOREM 3.1. For Lax-Wendroff with interpolation interface conditions, mesh refinement in time and space by any integer is stable.

Proof. To prove this, we must show that the root condition (2.9) cannot hold for $|z| \geqslant 1$. The idea of the proof is to apply the maximum principle (see, for example, Ahlfors, [1]) to the right-hand side of the root condition (2.9), call it $f(z)$,

$$
f(z)=\sum_{j=0}^{n-1}\left(\frac{z^{n}-1}{z-\bar{\omega}_{j}}\right)^{2} \frac{\bar{\omega}_{j}}{n^{2} z^{n-1}} \kappa_{j}^{n} .
$$

We will show that for $|z|>1$ there are no singularities, and that $f(z)$ is regular at $\infty$. It follows that the maximum modulus of $f(z)$ occurs for $|z|=1$. We know that $\tau$, the left-hand side of the root condition (2.9), satisfies $|\tau| \geqslant 1$. The last part of the proof consists of showing that $|f(z)| \leqslant 1-\delta, \delta>0$, for $|z|=1$, and so the root condition cannot hold.

First we check for branch points in $f(z)$ in the region $|z|>1$. This can only happen if there are branch points in the characteristic roots $\kappa$ and $\tau$ of Lax-Wendroff. From (2.2), this occurs for

$$
(z-1)^{2}+\lambda^{2}(2 z-1)=0 .
$$

This is a quadratic in $z$ with roots

$$
z_{ \pm}=\left(1-\lambda^{2}\right) \pm i \lambda \sqrt{1-\lambda^{2}}
$$

The modulus of the roots is

$$
|z|^{2}=\left(1-\lambda^{2}\right)^{2}+\lambda^{2}\left(1-\lambda^{2}\right)=1-\lambda^{2} \leqslant 1
$$

since for Cauchy stability for Lax-Wendroff, $0 \leqslant \lambda \leqslant 1$. Therefore, for $|z|>1$ there are no branch points.

Next we examine the expression $f(z)$ for large $z$, by checking the behavior of the $\kappa_{j}$ as $|z| \rightarrow \infty$. The characteristic equation (2.1) for Lax-Wendroff shows that the product of the two roots $\kappa_{\text {in }}$ and $\kappa_{\text {out }}$ satisfies

$$
\kappa_{\text {in }} \kappa_{\text {out }}=\frac{\lambda-1}{\lambda+1}
$$

with sum

$$
\kappa_{\text {in }}+\kappa_{\text {out }}=\frac{2\left(z+\lambda^{2}-1\right)}{\lambda^{2}+\lambda} .
$$


Furthermore, the root $\kappa_{\text {in }}$ stays inside the circle for $|z| \geqslant 1$. Otherwise, there would be a point where $\kappa_{\text {in }}$ crosses the circle, $\left|\kappa_{\text {in }}\right|=1$ with $|z|>1$, violating the von Neumann stability condition. Therefore, as $z \rightarrow \infty$,

$$
\kappa_{\mathrm{out}} \approx \frac{2 z}{\lambda^{2}+\lambda}
$$

and

$$
\kappa_{\mathrm{in}} \approx \frac{\lambda(\lambda-1)}{2 z} .
$$

Recalling that $\kappa_{j}=\kappa\left(z_{j}\right)$, and substituting (3.3) into (3.1) gives

$$
f(z)=\sum_{j} \frac{\bar{\omega}_{j}[(-\lambda)(1-\lambda)]^{n}}{2^{n} n^{2}} \frac{1}{z}+O\left(\frac{1}{z^{2}}\right),
$$

which is clearly bounded for large $|z|$.

Since $f(z)$ is regular at $\infty$ and has no branch points, the maximum modulus principle holds, which states that if $f$ is analytic in the interior and continuous up to the boundary of a region $R$, then the maximum modulus $|f(z)|$ occurs on the boundary. We will show that the maximum modulus of $f$ is less than, and bounded away, from 1 for $|z|=1$.

It is easy to show, as is done in Lemma 6.1 of Gustafsson et al., that the roots of Lax-Wendroff for $u_{t}=u_{x}$ satisfy

$$
\begin{aligned}
& \left|\kappa_{\text {in }}\right| \leqslant 1-\delta, \quad|z| \geqslant 1, \\
& \left|\kappa_{\text {out }}\right|>1, \quad|z| \geqslant 1, z \neq 1, \\
& \kappa_{\text {out }}=1, \quad z=1 .
\end{aligned}
$$

Therefore the root $\tau$ in (2.9) satisfies $|\tau| \geqslant 1$ for $|z| \geqslant 1$, and all the $\kappa_{j}$ satisfy $\left|\kappa_{j}\right| \leqslant 1-\delta$. We therefore have

$$
|f(z)| \leqslant(1-\delta)^{n} \sum_{j}\left|\rho_{j}\right| .
$$

By (2.3) we have that $\sum \rho_{j}=1$. Using $\left(z^{n}-1\right)^{2}=\left(z^{n}-1\right)\left(1-z^{-n}\right) z^{n}$ and the fact that $z^{-1}=\bar{z}$ for $z$ on the unit circle, the expression (2.8) for the $\rho_{j}$ can be rewritten

$$
\rho_{j}=\frac{1}{n^{2}} \frac{\left|z^{n}-1\right|^{2}}{\left|z-\bar{\omega}_{j}\right|^{2}} .
$$

Since the $\rho_{j}$ are real positive numbers, we have

$$
\sum_{j}\left|\rho_{j}\right|=1
$$

Therefore, in (2.9), the right-hand side $|f(z)| \leqslant(1-\delta)^{n}$, whereas the left-hand side $|\tau| \geqslant 1$. Thus the root condition cannot hold for $|z| \geqslant 1$, and so our approximation is stable.

We now assess the generality of the preceding methods. For general multi-level (in time) but three-point (in space) explicit difference schemes, the maximum principle still applies (Proposition 3.1). In fact, in the dissipative case, it is only necessary to check a finite number of $z$ values on the unit circle to determine stability. In the case 
of a two-level scheme, this can be done in general (Theorem 3.2). Note that the class of explicit dissipative three-point two-level schemes includes only Lax-Wendroff and various first-order schemes.

We show first that the maximum principle can still be applied. For $|z|>1$, the roots of the characteristic equation satisfy $\left|\kappa_{\text {in }}\right|<1<\left|\kappa_{\text {out }}\right|$, or else the von Neumann stability criterion would be violated. Thus (2.9) has no branch points for $|z|>1$. Next, the characteristic equation for an $s$ time-level scheme is

$$
\left(z^{s} Q_{-1}-\sum_{\sigma=0}^{s-1} z^{s-\sigma} Q_{\sigma}\right) \hat{v}=0 .
$$

For three-point explicit schemes, this gives a polynomial

$$
P(z, \kappa)=z^{s} \kappa+z^{s-1}\left(c_{0,-1}+c_{0,0} \kappa+c_{0,1} \kappa^{2}\right)+\cdots=0 .
$$

As $z \rightarrow \infty$, we must at least have $\kappa=O(1 / z)$. Since $\rho_{j}=O\left(z^{n-1}\right)$, the expression $f(z)=\sum_{j} \rho_{j} \kappa_{j}^{n}$ stays bounded. Thus, for general explicit three-point schemes, it is again necessary to check the root condition only for values of $z$ on the unit circle.

Since the root $\tau$ always satisfies $|\tau| \geqslant 1$, and the $\rho_{j}$ are real and positive with $\sum \rho_{j}=1$, we can only have $\tau=\sum_{j} \rho_{j} \kappa_{j}^{n}$ if $|\tau|=\left|\kappa_{j}\right|=1$, and in fact, if

$$
\tau\left(z^{n}\right)=\kappa_{j}^{n}\left(z \omega_{j}\right), \quad j=0, \ldots, n-1 .
$$

We summarize this in

Proposition 3.1. A three-point explicit finite-difference scheme (or combination of them) is unstable for mesh refinement in time and space with interpolation interface conditions if and only if it admits modes satisfying (3.6) for some $|z|=1$.

Various cases are now possible. If the coarse grid scheme is dissipative, the only $\operatorname{root}|\tau| \geqslant 1$ on the unit circle is $\tau=1$. If the scheme is two-level, by consistency this implies $z^{n}=1$. It is left to check the behavior of $\kappa_{j}=\kappa\left(z \omega_{j}\right), 0 \leqslant j \leqslant n-1$. If we use the same scheme on the fine grid as the coarse grid, and note that the root at $z=1, \tau=1$ is simple (see Goldberg and Tadmore, [11]), we must have $\kappa\left(z \omega_{j}=1\right)$ $\neq 1$ for some $j$. By dissipativity, $\kappa_{j} \neq 1$ implies $|z|<1$. Such an instability is therefore impossible, and we have the more general

THEOREM 3.2. Any two-level three-point explicit dissipative finite-difference scheme is stable for mesh refinement in time and space with linear interpolation at the interface.

Dissipativity guarantees that for a root $|\kappa|=1, \kappa \neq 1$, its amplification $|z|<1$. For multi-level dissipative schemes it is possible to have $\kappa=1$ for $z=1$ as well as other values $|z|=1$. Therefore, in our case it is possible to have the $\operatorname{root} \tau=1$ with $z^{n} \neq 1$. It is thus necessary to check stability for the finite number of values of $z$ for which $\tau\left(z^{n}\right)=1$. Goldberg and Tadmore exclude this case in their work by requiring the boundary scheme to satisfy the sufficient condition

$$
P(z, \kappa=1) \neq 0, \quad|z|=1, z \neq 1 .
$$

For three time-level schemes, Goldberg and Tadmor show that the only case to exclude is $z=-1$.

Finally, if only the fine grid scheme is dissipative, overall stability is still assured if condition (3.7) is satisfied. 
We make two remarks about these theorems. First, for dissipative schemes the stability results continue to hold for the equation $u_{t}=-u_{x}$. This is due to the separation of roots $\kappa, \tau$ inside and outside the unit circle. In a dissipative scheme, $|z|=1$ implies only one of $\kappa=1$ or $\tau=1$ holds, and the other root is off the circle. Thus, the root condition (2.9) cannot be satisfied. For nondissipative schemes, this is not necessarily the case. If Leapfrog is used to solve $u_{t}=u_{x}$, the parameters $z=1$, $\tau=1, \kappa_{0,1}= \pm 1$ yield an instability. However, if Leapfrog is used to solve $u_{t}=-u_{x}$, there is no instability for this mode, since for $z=1$, we now have $\tau=-1$. There is an asymmetry depending on whether the fine grid is on the upwind or downwind side of the interface.

The second remark concerns the case of unequal refinement factors in space and time. Our method of proof can be readily modified to cover this case as well. Let the fine grid be refined by a factor $n$ in time, but $m$ in space. The derivation of the constants $\rho_{j}, j=0, \ldots, n-1$, remains the same. The change comes from the final interface condition, which becomes $v_{1}^{0}=u_{m}^{0}$, or

$$
\tau=\sum_{j=0}^{n-1} \rho_{j} \kappa_{j}^{m}
$$

Our results for dissipative schemes continue to hold in this case. For mesh refinement in space only, $n=1$, we get the required matching condition $\tau=\kappa^{m}$. For mesh refinement in time only we get

$$
\tau=\sum_{j=0}^{n-1} \rho_{j} \kappa_{j}
$$

The only interesting thing in this case is that even the nondissipative Leapfrog scheme is stable, for $n>1$. As in (3.6), $\tau\left(z^{n}\right)=\kappa\left(z \omega_{j}\right), \forall j$, is necessary for instability. But for Leapfrog, the mapping between $z$ and $\kappa_{\text {in }}$ is one to one, and so the $\kappa_{j}$ are distinct. For the case $n=1,(3.8)$ degenerates to the transparent interface problem with no mesh refinement, which has a generalized eigenvalue.

4. Stability of the Coarse Mesh Approximation Method. We next consider the stability of an interface where the intermediate boundary values on the fine grid are determined by the Coarse Mesh Approximation Method (CMAM), instead of by interpolation in time. Recall from Section 2 the method of computing the coarse grid point at the interface: $v_{0}^{1}$ is computed using the fine grid point $u_{n}^{0}$ for the missing $v_{1}^{0}$. The CMAM uses this same method to compute the $u_{0}^{k}, 1 \leqslant k \leqslant n$. For the case of Lax-Wendroff, this is

$$
u_{0}^{k}=v_{0}^{0}+\frac{\Delta t_{k}}{2 h}\left(u_{n}^{0}-v_{-1}^{0}\right)+\frac{\Delta t_{k}^{2}}{2 h^{2}}\left(u_{n}^{0}-2 v_{0}^{0}+v_{-1}^{0}\right) .
$$

The time step $\Delta t_{k}=k \Delta t / n<\Delta t$, where $\Delta t$ is the time step on the coarse grid, so the boundary formulae are at least Cauchy stable. The advantage of this interface condition is that the equations are the same order of accuracy as the integration method, instead of the one order lower accuracy of linear interpolation. 
To examine the initial-boundary value stability of this configuration, we again look for modes

$$
\begin{array}{ll}
v_{\nu}^{m}=\left(z^{n}\right)^{m} \tau^{\nu}, & \nu=0,-1,-2, \ldots, \\
u_{\nu}^{m}=z^{m} \sum_{j=0}^{n-1} \rho_{j} \kappa_{j}^{\nu}\left(\omega_{j}\right)^{m}, & \nu=0,1,2, \ldots
\end{array}
$$

The condition $u_{0}=v_{0}$ gives

$$
\sum_{j=0}^{n-1} \rho_{j}=1
$$

The CMAM interface conditions (4.1) give

$$
z^{k} \sum_{j=0}^{n-1} \rho_{j} \omega_{j}^{k}=g_{k}, \quad k=1, \ldots, n-1,
$$

where $g_{k}$ is the amplification factor for the Lax-Wendroff operator with mesh ratio $k \lambda / n$,

$$
g_{k}=1+\frac{k \lambda}{2 n}\left(\tau-\tau^{-1}\right)+\frac{(k \lambda / n)^{2}}{2}\left(\tau-2+\tau^{-1}\right) .
$$

Again, we get the linear system

$$
\left(\begin{array}{ccccc}
1 & 1 & 1 & \cdots & 1 \\
\omega_{0} & \omega_{1} & \omega_{2} & \cdots & \omega_{n-1} \\
\omega_{0}^{2} & \omega_{1}^{2} & \omega_{2}^{2} & \cdots & \omega_{n-1}^{2} \\
\cdot & \cdot & \cdot & \cdot & \cdot \\
\omega_{0}^{n-1} & \omega_{1}^{n-1} & \omega_{2}^{n-1} & \cdots & \omega_{n-1}^{n-1}
\end{array}\right)\left(\begin{array}{c}
\rho_{0} \\
\rho_{1} \\
\rho_{2} \\
\cdot \\
\rho_{n-1}
\end{array}\right)\left(\begin{array}{c}
1 \\
g_{1} / z \\
g_{2} / z^{2} \\
\cdot \\
g_{n-1} / z^{n-1}
\end{array}\right)
$$

with solution

$$
\rho_{j}=\frac{1}{n} \sum_{k=0}^{n-1} \frac{g_{k} \bar{\omega}_{j}^{k}}{z^{k}}
$$

The root condition comes from substituting this expression for $\rho_{k}$ into the last interface condition, $v_{1}^{0}=u_{n}^{0}$, or equivalently $\tau=\sum_{j=0}^{n-1} \rho_{j} \kappa_{j}^{n}$, to get

$$
\tau=\frac{1}{n} \sum_{k=0}^{n-1} \sum_{j=0}^{n-1} g_{j}\left(\frac{\bar{\omega}_{k}}{z}\right)^{j} \kappa_{k}^{n}
$$

This double sum is a much more complicated expression than the root condition in Section 2. As before, we know that for values of $z$ with $|z| \geqslant 1,|\tau| \geqslant 1$. We would like to show that the right-hand side of (4.5) is always strictly less than 1. Again we use the maximum principle. To see that the right-hand side of (4.5) is bounded as $|z| \rightarrow \infty$, recall from Eq. (3.1) that $\kappa_{j} \approx-\lambda(1-\lambda) / 2 z_{j}$, so $\kappa_{j}^{n}$ decays like $z^{-n}$. This 
is needed to balance the growth of the terms $g_{k}$ in (4.4), which are functions of $\tau$. Recall that $\tau$ is the root of Lax-Wendroff outside the unit circle, $\tau^{-1}$ is inside, and this remains true for all $|z|>1$. As $z \rightarrow \infty$, from (3.2) we know $\tau \approx 2 z^{n} /\left(\lambda^{2}+\lambda\right)$, and so $g_{k}$ grows like $z^{n}$. Thus, for each term in the sum, the growth in $g_{k}$ is exactly cancelled by the decay in $\kappa_{j}$. This leaves the term $(1 / z)^{j}$, which only helps. Therefore, the maximum modulus of the right-hand side of (4.5) occurs for $|z|=1$, where (4.5) can be written

$$
\tau=\frac{1}{n} \sum_{j} \sum_{k} g_{j}{\overline{\left(\omega_{k} z\right)}}^{j} \kappa_{k}^{n} .
$$

This sum is difficult to work with analytically, and we turn directly to the computer to verify that for $|z|=1$ it has modulus less than 1 . Table 1 shows a typical calculation checking the magnitude for two refinement ratios $n=2$ and $n=4$, and three mesh ratios $\lambda=.2, \lambda=.5$, and $\lambda=.8$. In this table, we evaluate the right-hand side for 19 values of $z$ along the boundary of the unit circle; the modulus is nowhere close to 1 . Based on numerical experiments, we are convinced this holds for all mesh ratios $\lambda<1$.

\section{TABLE 1}

Modulus of right-hand side of (4.5) for several different refinement ratios $n$ and mesh ratios $\lambda$.

\begin{tabular}{|c|c|c|c|c|c|c|}
\hline$\theta$ & \multicolumn{2}{|c|}{$\lambda=.2$} & \multicolumn{2}{|c|}{$\lambda=.5$} & \multicolumn{2}{|c|}{$\lambda=.8$} \\
\hline$\left(z=e^{i \theta}\right)$ & $n=2$ & $n=4$ & $n=2$ & $n=4$ & $n=2$ & $n=4$ \\
\hline $.3142 \mathrm{e}+00$ & $.8337 e-01$ & $.6427 e-02$ & $.1087 e+00$ & $.1085 \mathrm{e}-01$ & $.1230 \mathrm{e}-01$ & $.1425 e-03$ \\
\hline $.6283 e+00$ & $.1637 e-01$ & $.1789 e-03$ & $.5754 \mathrm{e}-01$ & $.2516 \mathrm{e}-02$ & $.1148 \mathrm{e}-01$ & $.6516 e-04$ \\
\hline $.9424 \mathrm{e}+00$ & $.6110 e-02$ & $.1789 e-03$ & $.2020 \mathrm{e}-01$ & $.2516 \mathrm{e}-02$ & $.8706 e-02$ & $.6516 e-04$ \\
\hline $.1256 \mathrm{e}+01$ & $.2313 e-02$ & $.6427 e-02$ & $.7385 e-02$ & $.1085 \mathrm{e}-01$ & $.4980 \mathrm{e}-02$ & $.1425 e-03$ \\
\hline $.1570 \mathrm{e}+01$ & $.2042 \mathrm{e}-03$ & $.1975 e+00$ & $.1148 \mathrm{e}-03$ & $1236 e-01$ & $.2912 \mathrm{e}-02$ & $.1524 \mathrm{e}-03$ \\
\hline $.1885 \mathrm{e}+01$ & $.2313 e-02$ & $.6427 e-02$ & $.7385 e-02$ & $.1085 \mathrm{e}-01$ & $.4980 \mathrm{e}-02$ & $.1425 e-03$ \\
\hline $.2199 e+01$ & $.6110 e-02$ & $.1789 e-03$ & $.2020 \mathrm{e}-01$ & $.2516 \mathrm{e}-02$ & $.8706 e-02$ & $.6516 e-04$ \\
\hline $.2513 e+01$ & $.1637 e-01$ & $.1789 e-03$ & $.5754 \mathrm{e}-01$ & $.2516 \mathrm{e}-02$ & $.1148 \mathrm{e}-01$ & $.6516 e-04$ \\
\hline $.2827 e+01$ & $.8337 e-01$ & $.6427 e-02$ & $.1087 \mathrm{e}+00$ & $.1085 e-02$ & $.1230 \mathrm{e}-01$ & $.1425 \mathrm{e}-03$ \\
\hline $.3141 \mathrm{e}+01$ & $.4444 \mathrm{e}+00$ & $.1975 e+00$ & $.1111 \mathrm{e}+00$ & $.1236 \mathrm{e}-01$ & $.1234 \mathrm{e}-01$ & $.1524 \mathrm{e}-03$ \\
\hline $.3455 \mathrm{e}+01$ & $.8337 e-01$ & $.6427 e-02$ & $.1087 \mathrm{e}+00$ & $.1085 e-01$ & $.1230 \mathrm{e}-01$ & $.1425 e-03$ \\
\hline $.3769 e+01$ & $.1637 e-01$ & $.1789 e-03$ & $.5754 \mathrm{e}-01$ & $.2516 \mathrm{e}-02$ & $.1148 \mathrm{e}-01$ & $.6516 e-04$ \\
\hline $.4084 \mathrm{e}+01$ & $.6110 \mathrm{e}-02$ & $.1789 e-03$ & $.2020 \mathrm{e}-01$ & $.2516 \mathrm{e}-02$ & $.8706 \mathrm{e}-02$ & $.6516 e-04$ \\
\hline $.4398 \mathrm{e}+01$ & $.2313 e-02$ & $.6427 e-02$ & $.7385 e-02$ & $.1085 e-01$ & $.4980 e-02$ & $.1425 e-03$ \\
\hline $.4712 e+01$ & $.2042 e-03$ & $.1975 \mathrm{e}+00$ & $.1148 \mathrm{e}-03$ & $.1236 \mathrm{e}-01$ & $.2920 \mathrm{e}-02$ & $.1524 \mathrm{e}-03$ \\
\hline $.5026 \mathrm{e}+01$ & $.2313 e-02$ & $.6427 e-02$ & $.7385 e-02$ & $.1085 e-01$ & $.4980 \mathrm{e}-02$ & $.1425 e-03$ \\
\hline $.5340 \mathrm{e}+01$ & $.6110 \mathrm{e}-02$ & $.1789 e-03$ & $.2020 \mathrm{e}-01$ & $.2516 \mathrm{e}-02$ & $.8706 \mathrm{e}-02$ & $.6516 \mathrm{e}-04$ \\
\hline $.5654 \mathrm{e}+01$ & $.1637 e-01$ & $.1789 e-03$ & $.5754 \mathrm{e}-01$ & $.2516 e-02$ & $.1148 \mathrm{e}-01$ & $.6516 \mathrm{e}-04$ \\
\hline $.5969 e+01$ & $.8337 \mathrm{e}-01$ & $.6427 e-02$ & $.1087 \mathrm{e}+00$ & $.1085 e-01$ & $.1230 \mathrm{e}-01$ & $.1425 \mathrm{e}-03$ \\
\hline
\end{tabular}

It would be more satisfying to be able to prove stability analytically for all refinement ratios $n$ and mesh ratios $\lambda$. But, since we only need to check a single inequality for a smooth function of the one real variable $\theta$, the computer analysis is simple, quick, and we believe, as convincing as a complicated analytical proof. 
5. Overlapping Grids. In this final section we look at the modifications in the interface equations and stability conditions that occur when two grids do not meet exactly at a coordinate line. This mimics more closely the situation in two-dimensional calculations. If different coordinate systems are used in different parts of the domain (e.g., body-fitted coordinates around different parts of an airplane), it is simpler to have each grid end a short distance past the start of the other, rather than forcing a smooth transition from one system to another. Calculations using this principle have been successfully done by B. Kreiss [15] and Reyna [20]. Another example of this two-dimensional nonaligned interface is seen in Berger and Oliger, [4]. In their work on adaptive mesh refinement in time and space, they use refined grids which are rectangles rotated with respect to the coarse grid coordinate system. Again, spatial as well as temporal interpolation is used in the interface equations, since the boundaries of the fine and coarse grids do not coincide.

The situation we model in this case is the following.

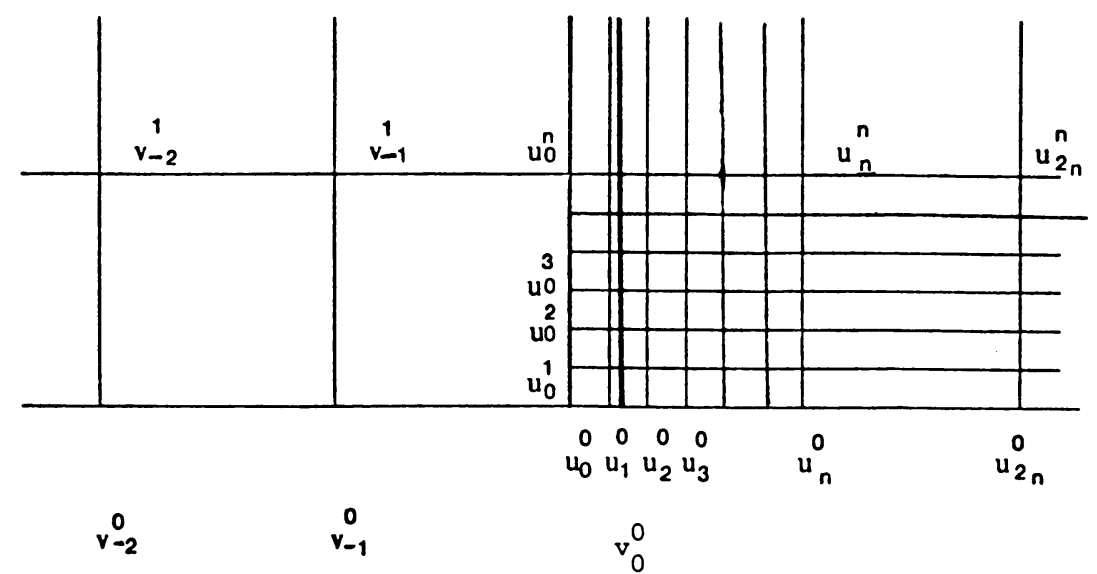

FIGURE 5.1

Overlapping grids with mesh refinement in space and time

In Figure 5.1, the fine and coarse grids overlap by a distance $d$. It is no surprise that if we use Lax-Wendroff to integrate the coarse and fine grids, we can prove that this overlapping mesh configuration is stable. Instead, we show that for the Leapfrog difference scheme, although the nonoverlapping case is unstable for the refinement ratio $n=2$ (Oliger, [17]), if the meshes overlap, the smoothing effect of linear interpolation is enough to stabilize the system.

We first make the small modifications needed in the root condition to account for the overlap. Proceeding as in Section 2, we look for modes $v_{\nu}^{m}=\left(z^{n}\right)^{m} \tau^{\nu}$ for $\nu \leqslant 0$, and $u_{\nu}^{m}=z^{m} \sum_{j=0}^{n-1} \hat{\rho}_{j} \kappa_{j}^{\nu}\left(\omega_{j}\right)^{m}$ for $\nu \geqslant 0$, where we use $\hat{\rho}_{j}$ for the constants now. The interface condition $u_{0}=v_{0}$ is no longer correct. Instead, we use linear interpolation in space to determine the fine grid value,

$$
u_{0}^{0}=v_{-1}^{0}+\frac{(h-d)}{h}\left(v_{0}^{0}-v_{-1}^{0}\right),
$$

along with linear interpolation in time,

$$
u_{0}^{k}=\frac{k}{n}\left(u_{0}^{n}-u_{0}^{0}\right), \quad 0<k<n .
$$


This gives the same linear system as before, but with a modified right-hand side,

$$
\begin{gathered}
\left(\begin{array}{ccccc}
1 & 1 & 1 & \cdots & 1 \\
\omega_{0} & \omega_{1} & \omega_{2} & \cdots & \omega_{n-1} \\
\omega_{0}^{2} & \omega_{1}^{2} & \omega_{2}^{2} & \ldots & \omega_{n-1}^{2} \\
\cdot & \cdot & \cdot & . & \cdot \\
\omega_{0}^{n-1} & \omega_{1}^{n-1} & \omega_{2}^{n-1} & \cdots & \omega_{n-1}^{n-1}
\end{array}\right)\left(\begin{array}{c}
\hat{\rho}_{0} \\
\hat{\rho}_{1} \\
\hat{\rho}_{2} \\
\cdot \\
\hat{\rho}_{n-1}
\end{array}\right) \\
=\left(\begin{array}{c}
\frac{1}{z}+\frac{1}{n} \frac{\left(z^{n}-1\right)}{z} \\
\frac{1}{z^{2}}+\frac{2}{n} \frac{\left(z^{n}-1\right)}{z^{2}} \\
\frac{1}{z^{n-1}}+\frac{n-1}{n} \frac{\left(z^{n}-1\right)}{z^{n-1}}
\end{array}\right)\left(\tau^{-1}+\left(\frac{h-d}{h}\right)\left(1-\tau^{-1}\right)\right) .
\end{gathered}
$$

As above, this system is easily solved and simplified to give, as before,

$$
\hat{\rho}_{j}=\frac{\left(z^{n}-1\right)^{2}}{\left(z-\bar{\omega}_{j}\right)^{2}} \frac{\bar{\omega}_{j}}{n^{2} z^{n-1}}\left(\alpha+(1-\alpha) \tau^{-1}\right)=\rho_{j}\left(\alpha+(1-\alpha) \tau^{-1}\right),
$$

where the $\rho_{j}$ are the constants from the previous nonoverlapping case in Section 2. Here we use $\alpha=(h-d) / h$.

The last interface condition specifies the value to use for $v_{1}^{0}$. It is set by interpolation from the fine grid. Let the underlying coarse point $v_{1}$ lie between fine points $u_{s}$ and $u_{s+1}$, so $s=n+\lfloor d n / h\rfloor$. We have

$$
v_{1}^{0}=(1-r) u_{s}^{0}+r u_{s+1}^{0},
$$

where $r$ is the distance from $v_{1}$ to $u_{s}, r=d-s h / n$, which means $0<r<1$. After substituting the normal modes into (5.3) we get the modified root condition

$$
\tau=\left[(1-r) \sum_{j} \rho_{j} \kappa_{j}^{s}+r \sum_{j} \rho_{j} \kappa_{j}^{s+1}\right]\left(\alpha+(1-\alpha) \tau^{-1}\right) .
$$

We point out that in the nonoverlapping case (take $r=0, \alpha=1$ and $s=n$ in (5.4)), the characteristic roots $\kappa_{1}=-1, \kappa_{2}=1$ and $\tau=1$ corresponding to $z=1$ are a solution of the root condition, and yield the generalized eigenvalue for the refinement ratio $n=2$.

To show there are no solutions of the root condition for $|z| \geqslant 1$, we again use the maximum principle, and therefore only check for solutions to (5.4) for $|z|=1$. The characteristic roots for Leapfrog satisfy the following estimates for $z=e^{i \theta}$ (Lemma 6.2 in Gustafsson, et al. [14]),

$$
\begin{array}{ll}
\left|\kappa_{\text {in }}\right|<1,\left|\kappa_{\text {out }}\right|>1 & \text { for }|\sin \theta|>\lambda, \\
\left|\kappa_{\text {in }}\right|=1,\left|\kappa_{\text {out }}\right|=1 & \text { for }|\sin \theta| \leqslant \lambda, \\
\kappa_{\text {in }}=-1, \kappa_{\text {out }}=1 & \text { for } \theta=0, \\
\kappa_{\text {in }}=1, \kappa_{\text {out }}=-1 & \text { for } \theta=\pi, \\
\kappa_{\text {in }}=\kappa_{\text {out }}= \pm i & \text { for } \sin \theta= \pm \lambda .
\end{array}
$$


The left-hand side of (5.4), $\tau$, satisfies $|\tau| \geqslant 1$. The factor $\alpha+(1-\alpha) \tau^{-1}$ on the right-hand side is always less than 1 , except when $\tau=1$. Since, using (3.4), the remaining part of the right-hand side is always less than or equal to 1 , it is only necessary to check for equality in the case $\tau=1$. Recall that $\tau$ is a function of $z^{n}=e^{i n \theta}$, which equals 1 only for $\theta=2 \pi j / n, j=0,1, \ldots, n-1$. For these values of $\theta$, we check whether

$$
(1-r) \sum_{j} \rho_{j} \kappa_{j}^{s}+r \sum_{j} \rho_{j} \kappa_{j}^{s+1}=1
$$

We can check this explicitly, using the representation (2.8) for $\rho_{j}$, and the fact that the $\rho_{j}$ 's sum to 1 . Let $z=\omega_{k}$. Then $\rho_{j}\left(z=\omega_{k}\right)=\delta_{j(n-k)}$, and so (5.5) becomes

$$
(1-r) \rho_{n-k} \kappa_{n-k}^{s}+r \rho_{n-k} \kappa_{n-k}^{s+1}=1 .
$$

Since $\rho_{n-k}=1$, it remains to check whether $\kappa_{n-k}=1$ for $z=\omega_{k}$. Looking at the characteristic equation for Leapfrog, and remembering that $\kappa_{n-k}$ is the root corresponding to the amplification in time $z \omega_{n-k}$, we get

$$
\kappa_{n-k}=\frac{\left(z \omega_{n-k}\right)^{2}-1}{2 \lambda\left(z \omega_{n-k}\right)}-\left(\frac{\left(1-\left(z \omega_{n-k}\right)^{2}\right)^{2}}{4 \lambda^{2}\left(z \omega_{n-k}\right)^{2}}+1\right)^{1 / 2} .
$$

It is easily determined that for $z=\omega_{k}, \kappa_{n-k}=-1$, and not 1 . Thus, there are no values $z$ and $\kappa$ for which the root condition (5.4) holds, and so Leapfrog with overlapping meshes and mesh refinement in time and space is stable.

We point out that we have not considered the case where the fine and coarse grids overlap by a distance $d$ where $d$ is a multiple of the fine mesh width $h / n$. This corresponds to taking $r=0$ in (5.5). In this case, spatial interpolation is not needed in computing $v_{1}^{0}$. Equation (5.5) reduces to determining if $\kappa_{n-k}^{s}=1$. One could presumably find values of $s$ and $n$ for which this holds. Reyna [20] has observed that straight injection at the interfaces in conjunction with Leapfrog is unstable in the case of mesh refinement in space only.

Acknowledgments. Thanks are due to Jonathan Goodman, Nick Trefethen, Eitan Tadmor and Joe Oliger for many helpful discussions.

Courant Institute of Mathematical Sciences

New York University

New York, New York 10012

1. L. Ahlfors, Complex Analysis, McGraw-Hill, New York, 1979.

2. M. Berger, Adaptive Mesh Refinement for Hyperbolic Partial Differential Equations, Ph. D. dissertation, Computer Science Dept., Stanford University, 1982.

3. M. Berger, On Conservation at Grid Interfaces, Icase Report No. 84-43, September, 1984.

4. M. Berger \& J. Oliger, "Adaptive mesh refinement for hyperbolic partial differential equations," J. Comput. Phys., v. 53, 1984, pp. 484-512.

5. J. Bolstad, An Adaptive Finite Difference Method for Hyperbolic Systems in One Space Dimension, Ph. D. dissertation, Computer Science Dept., Stanford University, 1982.

6. C. W. Boppe \& M. A. Stern, Simulated Transonic Flows for Aircraft with Nacelles, Pylons, and Winglets, AIAA Paper No. 80-0130, January 1980.

7. G. Browning, H.-O. Kreiss \& J. Oliger, "Mesh refinement," Math. Comp., v. 27, 1973, pp. 29-39.

8. M. Ciment, "Stable difference schemes with uneven mesh spacings," Math. Comp., v. 25, 1971, pp. 219-226. 
9. M. Ciment, "Stable matching of difference schemes," SIAM J. Numer. Anal., v. 9, 1972, pp. 695-701.

10. W. Coughran, On the Approximate Solution of Hyperbolic Initial Boundary Value Problems, $\mathrm{Ph} . \mathrm{D}$. dissertation, Computer Science Dept., Stanford University, 1980.

11. M. GOLDBERG \& E. TADMOR, "Scheme-independent stability criteria for difference approximations of hyperbolic initial-boundary value problems. II," Math. Comp., v. 36, 1981, pp. 603-626.

12. W. GROPP, “A test of moving mesh refinement for 2-D scalar hyperbolic problems," SIAM J. Sci. Statist. Comput., v. 1, 1980, pp. 191-197.

13. B. GuSTAFSSON, Proc. 1983 AMS-SIAM Summer Seminar on Large Scale Computations in Fluid Mechanics (La Jolla California), Lectures in Applied Math., vol. 22, Amer. Math. Soc., Providence, R. I., 1985.

14. B. GustafsSon, H.-O. Kreiss \& A. Sundstrom, "Stability theory of difference approximations for initial boundary value problems. II," Math. Comp., v. 26, 1972, pp. 649-686.

15. B. KreISS, "Construction of curvilinear grids," SIAM J. Sci. Statist. Comput., v. 4, 1983, pp. $270-279$.

16. H.-O. KREISS, "Stability theory for difference approximations of mixed initial boundary value problems. I,” Math. Comp., v. 22, 1968, pp. 703-714.

17. J. OlIGER, personal communication, 1979.

18. S. OSHER, "Systems of difference equations with general homogeneous boundary conditions," Trans. Amer. Math. Soc., v. 137, 1969, pp. 177-201.

19. V. Pereyra, W. Proskurowski \& O. Widlund, "High order fast Laplace solvers for the Dirichlet problem on general regions," Math. Comp., v. 31, 1977, pp. 1-16.

20. L. Reyna, On Composite Meshes, Ph. D. dissertation, Applied Math Dept., California Institute of Technology, 1983.

21. G. STARIUS, "On composite mesh difference methods for hyperbolic differential equations," Numer. Math., v. 35, 1980, pp. 241-255.

22. L. N. Trefethen, Wave Propagation and Stability for Finite Difference Schemes, Ph. D. dissertation, Computer Science Dept., Stanford University, 1982.

23. L. N. Trefethen, “Group Velocity Interpretation of the Stability Theory of Gustafsson, Kreiss and Sundström.” J. Comput. Phys., v. 49, 1983, pp. 199-217.

24. R. VICHNEVETSKY \& J. Bowles, Fourier Analysis of Numerical Approximations of Hyperbolic Equations, SIAM, Philadelphia, Pa., 1982. 